\title{
Politique
}

\section{Faut-il laisser notre sexe au vestiaire?}

\section{Diane Lamoureux et Micheline De Sève}

Numéro 15, hiver 1989

Paradigmes et scientificité

URI : https://id.erudit.org/iderudit/040617ar

DOI : https://doi.org/10.7202/040617ar

Aller au sommaire du numéro

Éditeur(s)

Société québécoise de science politique

ISSN

0711-608X (imprimé)

1918-6584 (numérique)

Découvrir la revue

Citer cet article

Lamoureux, D. \& Sève, M. D. (1989). Faut-il laisser notre sexe au vestiaire? Politique, (15), 5-22. https://doi.org/10.7202/040617ar d'utilisation que vous pouvez consulter en ligne.

https://apropos.erudit.org/fr/usagers/politique-dutilisation/ 


\title{
Faut-il laisser notre sexe au vestiaire?
}

\author{
Diane Lamoureux, \\ Université Laval \\ Micheline De Sève, \\ Université du Québec à Montréal
}

La science politique a-t-elle un sexe? Poser la question, c'est un peu y répondre. Car qui songe à y réfléchir sinon celles qui se sentent lésées par son sexisme? Plusieurs raisons peuvent nous amener à en conclure au sexisme; elles se résument cependant toutes dans celles-ci: en omettant de penser les rapports entre les sexes comme rapports de pouvoir, en occultant la dimension sexuée de ce dernier, la science politique est sexiste. Soit elle refuse de considérer les rapports de sexe comme rapports de pouvoir, les renvoyant à une infrapolitique qui sert de caution à un refus d'examen de la question ${ }^{1}$; soit elle refuse de considérer les femmes comme catégorie sociale d'analyse, les ravalant - au mieux - à un statut de variable ou d'exception à la règle,

1. Pourtant Aristote, peu suspect de féminisme, commence Le Politique par une analyse du pouvoir domestique. Ce n'est cependant que pour mieux montrer comment les rapports domestiques, parce que fondés sur la nécessité et non la délibération, ne relèvent pas du politique. 
masculine il va sans dire. Aussi banal que puisse être le constat, il est important de le rappeler puisque bon nombre de praticiens (et, hélas!, de praticiennes également) de la science politique refusent de l'admettre. La recherche des «coquilles» sexistes dans la plupart des manuels d'introduction à la discipline nous donnerait à elle seule un tableau de chasse bien garni.

Nous ne prendrons qu'un seul exemple, le Traité de science politique ${ }^{2}$, dont la première édition date de 1985 . Dans le premier tome, il est encore question de suffrage universel lorsqu'on veut parler de la disparition du cens électoral, comme si seuls les hommes participaient de l'universel. Plus loin, dans le débat sur la scientificité et l'objet de la discipline, il n'est aucunement fait mention des critiques soulevées par les féministes, les seuls interlocuteurs ayant droit à la parole dans ce débat étant des hommes, à quelques exceptions près. Par ailleurs, dans le tome 3 on néglige complètement l'apport des études féministes dans les débats sur la participation politique et le relatif renouvellement de ce champ d'analyse sous l'influence de chercheures féministes. Nous n'avons pas le temps d'entrer dans les détails, mais cela illustre le relatif aveuglement de la science politique par rapport à son sexisme.

Faut-il en déduire pour autant que la critique féministe est à l'origine d'une méthode féministe dans la discipline? Pas encore, dirions-nous, et nous irions même jusqu'à penser: surtout pas! Le féminisme nous semble un outil de critique sociale qui tire sa force du côté polymorphe de sa démarche. S'il opère par déconstruction, ce n'est pas pour substituer un nouveau savoir positif à celui en place. Le féminisme tire sa force d'une problématique, de sa volonté d'introduire partout un questionnement qui tienne compte de la réalité vécue par la moitié féminine de l'humanité. Mais cette moitié étant de facto aussi diversifiée que son pendant masculin, cela implique une réponse nécessairement non univoque aux questions posées.

Il s'agit précisément d'en découdre avec le simplisme réducteur d'une définition de l'éternel féminin qui exclurait les femmes du terrain

2. Ouvrage collectif sous la direction de Madeleine Grawitz et Jean Leca, paru au PUF, en 4 tomes. 
mouvant des affaires publiques. Nous voulons également attirer l'attention sur le fait que la politique du «diviser pour régner» fait fureur au plan de la déconsidération des courants critiques à l'intérieur de la discipline. Nous reviendrons sur ces questions à la fin du texte, mais d'ores et déjà, on peut dire que parler, actuellement, d'une approche féministe unifiée et systématisée en science politique relève, au mieux, du volontarisme et, au pire, de l'imposture.

Cependant, entre le volontarisme et le banal, il y a place pour des critiques féministes de certains aspects de la science politique, sans que toutefois nous prétendions à l'exhaustivité en la matière. Les points que nous avons choisi d'examiner sont ceux qui correspondent à notre pratique de la science politique. Ce ne sont pas nécessairement là les aspects les plus probants du sexisme dans la discipline même si la philosophie politique, pourtant si décriée - du fait de son caractère spéculatif - dans une discipline qui a un peu trop tendance à mesurer la scientificité à l'aune du mathématisable, sert de réservoir argumentaire de dernier recours. Par ailleurs, fidèles à notre point de vue critique, nous ne prétendons pas de cette manière poser les linéaments d'une philosophie politique non sexiste.

Ce que nous nous proposons de faire dans ce texte consiste donc à nous interroger sur l'objet de la science politique, pour montrer en quoi, dans la définition même de son objet, cette discipline tend à évacuer les femmes de son champ de réflexion. Dans un deuxième temps, nous aborderons la question de la scientificité et nous verrons l'utilisation qui peut être faite de cette notion pour discréditer la valeur de recherches entreprises sur la base d'une remise en cause des rapports sociaux de sexe.

\section{L'objet ou le déni des femmes}

La première chose qui frappe quiconque ouvre un manuel d'introduction à la science politique est certainement l'incertitude quant à son objet. Science du pouvoir ou science de l'État, ces deux réponses alimentent une polémique qui perdure depuis la naissance de la discipline. Par contre, au-delà de ces différences d'accent que nous ne cherchons nullement à minimiser, il y a un consensus qui s'est 
largement établi sur le fait que la science politique se préoccupe de phénomènes se déroulant dans ce qui est qualifié d'espace public. L'effet le plus évident d'un tel consensus, c'est d'évacuer les femmes d'une réflexion potentielle sur le politique.

Ce déni des femmes, la science politique l'a hérité de la philosophie politique dont elle s'est tellement souciée de se démarquer par ailleurs. Et la tradition est ancienne. Nous n'avons pas le projet de brosser un panorama complet de l'histoire de la pensée politique, mais il est tout de même utile d'examiner comment, à travers cette pensée, se construit l'exclusion des femmes. À tout seigneur, tout honneur, commençons donc par Aristote dont tous les manuels nous racontent qu'il est le grand ancêtre de la discipline.

Dans Le Politique, Aristote commence par cerner son objet et entreprend de distinguer le lien politique des autres liens sociaux. Il distingue donc les liens fondés sur la nécessité et ceux fondés sur la liberté. Dans les liens nécessaires, il classe la famille, dans son sens productif et reproductif, et il en fait une structure nécessairement hiérarchique puisque «toute famille, en effet, est régie dans la forme monarchique par le mâle le plus âgé» ${ }^{3}$. Par ailleurs, la cité, même si elle constitue une forme de regroupement naturelle, est de l'ordre de la liberté puisqu'elle permet aux hommes de se distinguer des autres animaux, ce qui l'amène à soutenir que «l'homme est par nature un animal politique. Et celui qui est sans cité, naturellement et non par suite des circonstances, est ou un être dégradé ou au-dessus de l'humanité» ${ }^{4}$. Ensuite, il suffit d'exclure la famille du champ de la réflexion politique pour en exclure les femmes.

Cette tradition inauguré par Aristote a la vie longue. Elle sera reprise dans la pensée politique moderne par Rousseau, dont l'admiration pour l'Antiquité le conduira à délimiter très strictement et à un niveau rarement aussi explicite avant lui, l'espace privé et l'espace public. Le «cher Jean-Jacques», par ailleurs si sensible à l'inégalité sociale, ne peut imaginer entre les sexes autre chose qu'une stricte répartition des sphères d'activités qui fera fureur en sociologie de la

3. Aristote, Le Politique I, II, (Les citations sont extraites de l'édition Vrin 1987, traduction F. Tricot), p. 26.

4. Ibid., p. 28. 
famille: aux femmes, l'affectif, aux hommes, l'instrumental! Toute sa pensée concernant les femmes relève d'ailleurs de ce qu'on appelle maintenant le «double standard»:

"Dès qu'une fois il est démontré que l'homme et la femme ne sont ni ne doivent être constitués de même, de caractére ni de tempérament, il s'en suit qu'ils ne doivent pas avoir la même éducation. En suivant les directions de la nature ils doivent agir de concert, mais ils ne doivent pas faire les mêmes choses; la fin des travaux est commune, mais les travaux sont différens et par consequent les gouts qui les dirigent ${ }^{5}$.

Ainsi, la force physique, qui ne peut se justifier d'aucun fondement rationnel dans les relations entre les hommes et qui, par conséquent, ne peut être invoquée pour fonder la domination d'un groupe d'hommes sur un autre, trouve-t-elle à s'employer dans le cadre des relations familiales. En effet, ce qui justifie le statut subordonné des femmes à l'intérieur de la famille, c'est la nécessité dans laquelle elles sont de se trouver un protecteur, puisqu'elles «dépendent des hommes et par leurs désirs et par leurs besoins» ${ }^{6}$. N'ayant pas de liberté personnelle, elles sont donc exclues de la formation de la volonté générale. Pourtant, Rousseau confère au contrat social le rôle de suppléer aux inégalités naturelles et de créer un statut d'isonomie. Mais son champ d'application présente des limites...

De même, la famille lui apparaît comme une institution purement naturelle, et non sociale, et les rapports inégalitaires entre les sexes reposent sur la raison et sa capacité de décoder les lois de la nature ${ }^{7}$. Ce qui résulte logiquement de l'idée que la force est à la base des relations entre les sexes. Cependant, il situe le fondement de la

5. Jean-Jacques Rousseau, Émile, Oeuvres complètes, tome IV, p. 700. Les références à Rousseau sont tirées de l'édition de la bilbiothèque de la Pléiade.

6. Ibid., p. 702.

7. Cf. Émile, p. 697. «... cette inégalité (entre les sexes) n'est point une institution humaine, ou du moins elle n'est point l'ouvrage du préjugé mais de la raison». 
famille non dans la force, mais dans le sentiment. N'ayant pas de volonté propre, les femmes ne peuvent participer à une institution sociale. Par ailleurs, en fait de sentiment, Rousseau nous parle plus de sa peur des femmes que de la sentimentalité féminine. Réduite à un rôle d'influence politique, les femmes se doivent d'être chastes et vertueuses afin de rassurer leur mari sur la «légitimité» de leur progéniture. La démocratie qu'il préconise devient donc la démocratie des chefs de famille.

On en trouve confirmation dans le commentaire qu'il fait, dans le livre V de l'Émile, sur l'utopie platonicienne. Il lui reproche à la fois la disparition de la famille, et la trangression des rôles sexuels ce qui ne laisse d'autre choix à Platon que d'hominiser les femmes:

"Platon dans sa République donne aux femmes les mêmes exercices qu'aux hommes; je le crois bien! Ayant ôté de son gouvernement les familles particulières et ne sachant plus que faire des femmes, il se vit forcé de les faire hommes. Ce beau génie avoit tout combiné, tout prévu: il alloit au devant d'une objection que personne n'eut songé à lui faire, mais il a mal résolu celle qu'on lui fait. Je ne parle point de cette prétendue communauté des femmes (...) je parle de cette promiscüité civile qui confond partout les deux sexes dans les mêmes emplois, dans les mêmes travaux et ne peut manquer d'engendrer les plus intolérables abus: je parle de cette subversion des plus doux sentimens de la nature, immolés à un sentiment artificiel qui ne peut subsister que par eux; comme s'il ne faloit pas une prise naturelle pour former des liens de convention; comme si l'amour qu'on a pour ses proches n'étoit pas le principe de celui qu'on doit à l'État; comme si ce n'étoit pas par la petite patrie qui est la famille que le coeur s'attache à la grande; comme si ce n'étoitent pas le bon fils, le bon mari, le bon père qui font le bon citoyen ${ }^{8}$.

L'idée rousseauiste d'une différence entre les sexes qui engendre une ségrégation des espaces sociaux allait inspirer les révolutionnaires français dans leur refus d'accorder aux femmes le droit de cité. Malgré

8. Ibid., pp. 699-700. 
des tentatives de formuler, dans le sillage des droits de l'homme, les droits des femmes ${ }^{9}$, c'est la différence des rôles sociaux entre les sexes qui allait primer et il faudra un long et patient mouvement suffragiste pour que les femmes puissent apparaître comme électrices dans le champ politique. Cette idée d'une "démocratie sans les femmes» ${ }^{10}$ allait également imprégner la pensée de Tocqueville:

"On peut aisément concevoir qu'en s'efforçant d'égaler ainsi un sexe à l'autre on les dégrade tous les deux; et que dans ce mélange grossier des oeuvres de la nature il ne saurait jamais sortir que des hommes faibles et des femmes déshonnêtes. (...) L'Amérique est le pays du monde où l'on a pris le soin le plus continuel de tracer aux deux sexes des lignes d'action nettement séparées, et où on a voulu que tous deux marchassent d'un pas égal, mais dans des chemins toujours différents. Vous ne voyez point d'Américaines diriger les affaires extérieures de la famille, conduire un négoce, ni pénétrer enfin dans une sphère politique...11».

Quant à Marx, son discours sur les femmes est encore plus ambigu. On connaît son mépris des droits politiques, qu'il ravale au rang d'illusion ${ }^{12}$. De plus, malgré son indignation, commune aux petits-bourgeois de l'époque, devant le travail des femmes et des enfants, il définit l'humanité par le travail ${ }^{13}$. Cependant, et cela est un aspect crucial en ce qui concerne les femmes, sa définition du travail est des plus restrictives. Ni la gestation/lactation, ni le travail ménager

9. Nous pensons ici principalement à Les droits de la femme et de la citoyenne d'Olympe de Gouges et à la Défense des droits de la femme de Mary Wollstonecraft.

10. Nous empruntons cette expression à Christine Fauré qui en a fait le titre d'un ouvrage publié aux PUF en 1985.

11. Alexis de Tocqueville, De la démocratie en Amérique, tome II, Bouquins-Laffont, 1986, pp. 572-573. $10 / 18$.

12. Cf. La question juive, principalement pp. 36-41 de l'édition

13. Cf. Le Manifeste du parti communiste, ou encore L'idéologie allemande. 
ne constituent un travail socialement productif ${ }^{14}$. Or, son schéma d'émancipation du/dans le travail repose justement sur le travail socialement productif.

Ce refus de penser dans son entièreté la notion de travail pour se concentrer sur le seul travail productif ne permet pas à Marx d'élaborer un projet d'émancipation sociale sexuellement non connoté. Certes, dans leur dimension de «travailleuses productives», les femmes sont incluses à égalité avec les hommes dans le projet socialiste. Mais il y a tout le refoulé du travail ménager et procréatif qui les retient de participer de plein droit à l'émancipation des travailleurs.

Ce survol rapide nous permet de constater qu'il ne suffit pas d'inclure les femmes au nombre des détenteurs des droits politiques pour désexiser la pensée politique. Celle-ci a une longue tradition reposant sur une dichotomie public/privé qui s'avère cruciale. De fait, le monde public présuppose l'existence d'un privé maîtrisé. Cela nous aide à mieux comprendre pourquoi l'extension des droits politiques aux femmes dans la première moitié du $20 \mathrm{e}$ siècle a été suivie de peu de conséquences pratiques. Il faudra attendre que l'accès à la contraception se généralise pour que l'identité sexuelle des femmes cesse de se représenter quasi-exclusivement sous l'angle de la contrainte à la maternité. Le contenu de leurs activités est ainsi devenu l'enjeu de décisions politiques dont on sait l'impact qu'elles exercent sur l'évolution démographique et la qualité de la vie. Mais là n'est pas notre propos...

Il faut voir cependant que cette mise des femmes «hors-champ» du regard politique persistera dans le passage de la philosophie à la science politique. Nannerl $O$. Keohane attribue cet oubli des femmes principalement à deux facteurs. D'une part, la science politique, confrontée dans ses débuts à la non-participation politique des femmes

14. Nous n'avons pas l'intention de reprendre ici, pour en ennème fois le débat sur la production domestique, référons les lectrices et lecteurs intéressés à l'ouvrage de Mary O'Brien, The Politics of Reproduction, Londres, Routledge and Kegan Paul, 1981 (traduction française, La dialectique de la reproduction, Montréal, Éditions du remueménage, 1987), et à celui sous la direction de Louise Vandelac, $D u$ travail et de l'amour, Montréal, Saint-Martin, 1986. 
du fait de leur non-appartenance à la communauté politique, n'est pas parvenue à forger des instruments adéquats pour comprendre le pouvoir informel des femmes, ce qui a conduit à une espèce d'invalidation de l'«éminence rose» puisque «students of politics have taken refuge in mutterings about inscrutinability, irrationality, or hormones, and dismissed the power of womankind as an unpredictable force that has no place in a properly ordered political universe ${ }^{15}$. D'autre part, la science politique a ignoré les diverses façons dont peut s'exercer le pouvoir en concentrant son attention sur les institutions étatiques, ce qui a eu pour conséquence que «the study of politics has systematically neglected those arenas in which women are most frequently found in authoritative roles» $^{16}$.

Ce n'est que très récemment, et sous l'influence de féministes politologues dans la grande majorité des cas, que la science politique a commencé à faire entrer les femmes dans son champ de vision. Mais pour l'instant la tentative est encore timide et ne jouit d'une certaine légitimité scientifique que dans le champ de la participation politique. Du fait qu'il y a maintenant un nombre croissant de femmes qui exercent des fonctions politiques électives ou administratives et qu'on ne peut donc désormais nier leur présence dans la sphère publique stricto sensu, du fait également de la résurgence d'un féminisme qui, à certaines occasions, a rempli les fonctions classiques d'un groupe de pression, les femmes peuvent maintenant constituer un objet d'étude pertinent pour les catégories d'analyse traditionnelles de la science politique. Mais il ne semble pas pour autant que la science politique soit prête à faire véritablement place à l'idée que le personnel soit politique et à une remise en cause de la dichotomie entre sphère publique et sphère privée, ce qui constitue, à plusieurs égards, le défi majeur que lance le féminisme à la science politique.

15. Nannerl O. Keohane, «Speaking from silence», Elisabeth Lange et Walter Gove (ed.) A Feminsit Perspective in the Academy, Chicago University Press, 1981, p. 89.

16. Ibid., p. 89. 


\section{Le scientifique et le politique}

Ceci nous amène à notre deuxième question, qu'est-ce que la science dans la politique, c'est-à-dire comment arriver à des approximations du vrai à partir d'une réflexion sur la politique? On peut également se poser la question suivante: peut-on être féministe et faire de la science politique au sens de la respectabilité académique du terme? Il est difficile d'y répondre pour deux raisons. D'une part, comme nous l'avons déjà mentionné, le consensus est loin d'être établi, dans la discipline, sur ce qui constitue l'objet de la science politique et cela a des conséquences directes sur la «visibilité» des femmes. D'autre part, le statut de la critique épistémologique y est également problématique.

Nous poursuivrons le raisonnement entrepris dans la première partie de notre réflexion en essayant, maintenant, de cerner ce que peut receler l'expression «le personnel est politique». Certains sens en ont rapidement été explorés et nous n'avons pas l'intention de nous y attarder. Ainsi, il est maintenant d'usage d'admettre que nombre d'aspects de la vie des citoyens et citoyennes sont encadrés par l'action de l'État: en effet, celui-ci régit les relations interpersonnelles en légiférant sur le mariage, le divorce, les obligations et responsabilités des parents à l'égard des enfants, etc. De plus, dans la foulée des mouvements anti-autoritaires de la fin des années ' 60 , il a été de mise de traquer le pouvoir partout où il s'exerçait, y compris dans les aspects les plus intimes de nos vies, créant ainsi une dynamique du «tout est politique». Mais il nous semble qu'il est désormais possible, suite à l'expérience de la dernière vague du féminisme, celle des années '70, d'opérer une autre lecture des rapports entre personnel et politique.

Nous articulerons notre réflexion à celle de Françoise Collin qui souligne que:

«Le féminisme est le premier mouvement à poser la question politique par excellence, celle de l'absence de droits dans un État de 
droit. Et c'est fondamentalement la notion de liberté qui le travaille, plus que celle de l'égalité comme égalisation ${ }^{17}{ }^{\star}$.

En fait, ce que le féminisme contemporain a permis de mettre en lumière c'est que, dans nos sociétés, les femmes ne sont pas encore considérées comme des individus. Dans ce sens, ce qui apparaît à première vue comme revendication de l'égalité, par exemple, le droit à l'avortement, l'égalité des droits civils, l'équité en emploi, la protection légale contre la violence «privée» (viol, violence conjugale, harcèlement sexuel, pornographie) peut également être entrevu comme une démarche d'individuation au sens où Macpherson définissait l'individu libéral «as an owner of himself (...) The human essence is freedom from the dependance on the will of others ${ }^{18}$. Ainsi, il conviendrait de parler de contrôle de son corps, de lutte contre la puissance maritale, d'accès autonome aux ressources matérielles et du droit à la sûreté garanti par l'État.

La réflexion de Collin se poursuit en insistant sur le caractère à la fois moderne et post-moderne du mouvement féministe, soulignant qu'on aurait tort de l'assimiler à un simple mouvement social «instituant les femmes comme genre, animé par une volonté supposée générale. Ainsi le pernicieux concept de sororité est-il venu accentuer les ambiguittés du concept de fraternité, barrant la voie au dialogue au nom d'un unanimisme postulé» ${ }^{19}$. Cela permet d'aborder le rapport du mouvement des femmes au politique. Mouvement social, il l'a été dans la mesure où il a pensé que par des luttes dans la société civile, on pouvait parvenir à contourner l'État. Mais en même temps, il n'a pu faire l'économie d'une réevaluation de ses rapports avec l'État, l'ambiguitté de son rapport à l'étatique constituant même une des causes essentielles de sa léthargie actuelle. Cela se fait sentir simultanément sur trois plans: d'abord, il a été partiellement happé par les institutions; ensuite, ses succès on fait voler en éclat, si tant est

17. Françoise Collin, «Introduction», Cahier $d u$ GRIF, $\mathrm{n}^{\circ} 33$, 1986, p. 6.

18. C.B. Macpherson, The Political Theory of Possessive Individualism, Oxford University Press, 1985, p. 3.

19. Françoise Collin, op. cit. 
qu'elle ait jamais existé, l'homogénéité de la «condition féminine»; enfin, parce qu'il est toujours à la recherche de modes de règlement de ses différends internes, oscillant entre l'unanimisme et la paralysie.

Mais en même temps, le féminisme a été le lieu politique par excellence des femmes si nous nous en tenons à la définition aristotélicienne, reprise par Arendt, du politique, à savoir le lieu de la confrontation et de la prise de parole. L'apparition des diverses vagues féministes prend toujours la forme d'une explosion du verbe. L'acte premier est celui de nommer et de créer un monde commun par le langage. Qui peut nous assurer que la violence conjugale, le viol, l'avortement auraient été considérés comme des enjeux sociaux si le féminisme, par le biais d'abord des groupes de conscience, ne les avait fait sortir du statut d'expériences personnelles pour en dévoiler les ressorts sociétaux? De même, le féminisme aura été le lieu de l'«agon» aristotélicien, ce qui explique l'âpreté de certaines polémiques.

Une telle compréhension de la notion «le personnel est politique» nous amène donc à remettre en cause la dichotomie entre sphère privée et sphère publique. Cette partition binaire de la sphère sociale nous semble relever d'une pensée de la domination, hiérarchisante et mutilante. Ce qui soulève un problème de taille puisque le mode binaire de raisonnement caractérise la pensée occidentale et que, au moins depuis Descartes, binarité et rationalité semblent s'équivaloir. Et si le problème n'était pas assez important, on peut toujours y ajouter le rapport entre modernité et rationalité. Bref, sous des dehors anodins, ce à quoi nous conduit «le personnel est politique», c'est à une entreprise de déconstruction de la rationalité occidentale $^{20}$. Nous allons la laisser en plan pour le moment...

Nous passerons donc à la deuxième question que nous soulevions, celle de la «science» dans la discipline. Pour ce faire, nous partirons de la distinction weberienne entre le «savant» et le

20. C'est là un élément qu'a très bien vu Habermas et qui soutient ses tentatives de défense de la modernité par rapport aux discours de la déconstruction post-moderne. Voir à ce propos deux ouvrages principalement, Théorie de l'agir communicationnel et Le discours philosophique de la modernité. 
«militant» ${ }^{21}$ et de sa notion de neutralité axiologique pour voir quel est le statut réservé à la critique féministe dans ce domaine. Rappelons que pour Weber, le savant est l'analyste neutre (et ajouterions-nous, asexué) des processus politiques dans lesquels il n'aurait aucun intérêt particulier autre qu'heuristique, ce qui lui permettrait d'analyser rationnellement les effets probables de la combinaison des composantes de la conjoncture, alors que le militant serait l'acteur par excellence, possiblement pourvu d'une solide connaissance du réel, mais dont toute l'énergie serait tendue vers les processus de consolidation et de transformation politiques.

Cette distinction a largement été utilisée pour tenter de dévaluer les courants critiques à l'intérieur de la discipline, principalement ceux qui se réclamaient de leurs liens avec des mouvements sociaux. Cela avait en outre l'avantage de dédouaner ce qu'on pourrait appeler le «courant dominant» ou, plus exactement, ce qui se donne à voir comme tel. Peut-on avoir un regard «neutre» qui soit autre que celui du vainqueur et qui ne fonde en réalité sa «neutralité» sur sa domination? Ce problème avait d'ailleurs déjà été entrevu par Weber qui mettait en garde contre la supposée neutralité des faits ${ }^{22}$. Bref, nous dirions que, contrairement au «courant dominant» la critique féministe procède d'un point de vue partial qui ne cache point sa partialité, ce qui ne l'empêche aucunement d'être rigoureux ${ }^{23}$.

En fait, nous pourrions faire nôtre une mise en garde de Weber qui précise que:

«La conception à combattre dans tous les cas est celle qui considère que, du point de vue scientifique, on pourrait se contenter de l'évidence effective, consacrée par une convention, de certaines prises

21. Cf. Max Weber, Le Savant et le politique, Paris, UGE, 10/18, 1979.

22. Voir ses Essais sur la théorie de la science, Paris, Plon, 1965 et plus particulièrement l'article «Le sens de la neutralité axiologique».

23. Cf. Danielle Juteau-Lee, «Visions partielles, visions partiales: visions (des) minoritaires en sociologie» dans Sociologie et Sociétés, vol. $13, \mathrm{n}^{\circ} 2,1981$. 
de position pratiques, si répandues soient-elles. La science me semble remplir une fonction spécifiquement inverse: elle fait de ce qui est évident par convention un problème $e^{24}$.

Ce que nous cherchons justement à faire, c'est de problématiser les «évidences» et une des premières à laquelle nous nous heurtons est celle de l'invisibilité politique des femmes. Il va de soi que l'objectivité, ce pourrait être de rétablir l'équilibre en introduisant à côté de la catégorie homme, une catégorie femme sans laquelle toute prétention à l'universel est illusoire. C'est d'ailleurs l'interprétation qui a permis de dénoncer le caractère sexiste des recherches traditionnelles en science politique ${ }^{25}$. L'objectif d'une telle démarche est d'imposer l'élargissement des catégories d'analyse à la variable femme dans toute recherche digne de ce nom. Mais il y a là un piège.

En effet, a contrario, cela impose d'axer aussi les recherches féministes en général sur le rapport hommes-femmes, ce qui pourrait avoir pour effet de marginaliser sinon discréditer toutes les recherches qui prétendraient poursuivre l'exploration de l'espace politique offert par les relations des femmes entre elles, terra incognita s'il en est. C'est un peu comme si, au nom de l'idée de l'État canadien, on entendait condamner comme dorénavant dépassée toute recherche exclusivement consacrée à l'une ou l'autre province, ce qui aurait eu pour effet de paralyser assez sérieusement, avouons-le, les études québécoises. Il convient au contraire d'encourager l'exploration d'un domaine nouveau de connaissance et de dégager un maximum de ressources pour combler un retard historique qui n'a malheureusement rien d'accidentel. Certes, les études sur les femmes sont partielles, mais tant qu'elles ne prétendent pas à l'universalité, il n'y a aucune raison de mettre en doute leur scientificité ou encore leur pertinence. En outre, ce type d'argument relève de l'hétérosexisme: comme si les femmes n'avaient de sens que rapportées aux hommes! p. 419 .

24. Max Weber, «Le sens de la neutralité axiologique», op. cit.,

25. Margrit Eichler et Jeanne Lapointe, Le traitement des sexes dans la recherche, Conseil de recherches en sciences humaines, Ottawa, 1985. 
Dans l'état actuel des choses, nous obliger à procéder systématiquement par référence ou par comparaison à un groupecontrôle mâle, c'est ralentir sérieusement une démarche dont le moins qu'on puisse dire est qu'elle n'a pas les moyens de se disperser de la sorte sur le plan conceptuel, c'est d'autant plus grave que cela risque d'inhiber la formulation de concepts adaptés à la réalité du monde politique des femmes comme êtres sociaux à part entière indépendamment de leur rapport à l'autre moitié du genre humain:

«The rejection of the male-as-norm principle provides a basis for a reassesment and evaluation of women's political potential and their political input that asserts them as political beings equal to $m^{26}{ }^{26}$.

Cela rend également beaucoup plus difficile la réflexion autour de l'autonomie des femmes. Tant que le discours féministe se contentait d'osciller entre la quête de l'égalité et la revendication de la différence, il restait à l'intérieur de cadres bien balisés et somme toute relativement digestibles de la pensée politique. À partir du moment où le féminisme, dans la pratique, se réclame de l'autonomie des femmes et se refuse au détour obligé par le référent masculin et où des chercheures féministes en font autant dans le domaine théorique, les affaires se corsent. Nous assistons actuellement, et cela se pare souvent, de façon ironique, des oripeaux de l'égalité entre les sexes, à une offensive en règle contre cette autonomie dans laquelle les institutions voient, à juste titre, une menace importante.

Il arrive trop souvent que des projets de recherche féministes soient rejetés par de savants comités de pairs comme «idéologiques» par suite du strict découpage de leur objet. La méfiance des milieux intellectuels envers la recherche critique ou la recherche-action est ici poussée d'un cran; il ne s'agit plus seulement d'un jugement prétendant exclure la recherche féministe du monde de la science au même titre que toute autre recherche engagée mais d'une décision à caractère

26. Maria Markus, «Deconstructed Inequalities: Women and the Public Sphere», Thesis Eleven, 14, 1984, p. 126. 
politique visant à inscrire la recherche sur les femmes - et elle seule - dans une démarche comparative. Le prétexte de recherche nonsexiste peut ainsi servir à évincer des recherches spécifiques qui commencent à peine à nommer l'univers-femme et ce, indépendamment de leur position plus ou moins radicale dans le champ des études féministes. Ce qui est sexiste, soulignons-le une fois de plus.

Prenons garde qu'une fois encore l'introduction de règles destinées à nous "protéger» en condamnant le sexisme des recherches en sciences humaines serve à discréditer le courant fragile des recherches féministes uniquement soucieuses de dire l'espace politique des femmes entre elles ${ }^{27}$. De fait, quand nous nous élevons contre toute prétention à proposer un cadre de recherche unique, une méthode unique à la recherche féministe, c'est précisément parce que nous craignons que, chassé par la porte, l'androcentrisme ne revienne par la fenêtre. N'est-ce pas le cas des écoles qui établissent une hiérarchie entre recherches quantitatives dites «scientifiques» et recherches qualitatives, jugées «pré» ou «a-scientifiques»? Là encore, la querelle n'est pas innocente qui oppose un secteur constitué, appuyé sur des statistiques et des données systématiquement répertoriées depuis des décennies, et un champ encore en friche, à la recherche de ses hypothèses sur un terrain où tant d'éléments restent à repérer et à baliser.

Plus encore que le sexisme, ce qu'il importe de mettre en lumière, c'est l'androcentrisme de la discipline. Il est certes faisable, et l'expérience a permis de le vérifier dans certains domaines d'analyse, d'intégrer la réalité des femmes comme variable ou encore fluctuation par rapport à un modèle. À cet égard, les études sur la participation politique des femmes se sont avérées particulièrement fructueuses. Mais ce qu'elles nous démontrent aussi, c'est la déficience de modèles élaborés à partir de la seule expérience des hommes. Une science politique non-sexiste, se devra donc de rejeter l'androcentrisme, c'est-à-

27. Voir là-dessus le passionnant débat entre Jill Vickers et Margrit Eichler dans RFR-DRF, Documentation sur la recherche féministe, vol. 16, $\mathrm{n}^{\circ} 4$ 1987, pp. 3-6. 
dire la valorisation de la seule expérience masculine et son érection en modèle universellement valable.

Dans le contexte actuel de la production et de la diffusion des connaissances scientifiques, l'expérience des femmes est encore discréditée comme plus particulariste que celle des hommes. La mesure de l'expérience masculine du rapport au monde reste encore trop souvent le critère obligé de passage à l'universel. L'entreprise de réhabilitation des femmes comme êtres sociaux à part entière passe par la déconstruction de ce schéma mental où le mot «homme» peut apparaître comme un terme générique alors que le mot «femme» n'est qu'un terme spécifique. L'un des moyens pour y parvenir, c'est d'accorder aux femmes la liberté de construire à leur tour leurs propres définitions sans autre contrainte que leurs propres visions du monde. Procéder par comparaison ne saurait suffire puisque cela s'inscrit dans une logique où les femmes doivent passer par le référent à la position de l'autre sexe - ajuster leurs perceptions aux siennes - pour se situer politiquement alors que les hommes, dans le meilleur des cas, se contentent d'introduire quelques nuances sans pour autant redéfinir leurs catégories d'analyse ni cesser d'occuper une position dominante tant dans la production du discours scientifique qu'aux commandes de l'État.

Ce qui fait que, pour l'instant, l'éclectisme est encore préférable à un dogmatisme qui sous couleur de définir «les aspects codifiables de la recherche féministe» ${ }^{28}$ pourrait préluder à une forme de récupération par les pouvoirs en place. Faute d'arriver à effacer notre présence, ce compromis destiné à nous réconcilier avec l'environnement social et intellectuel dominant ${ }^{29}$ ne servirait qu'à reproduire ad nauseam notre secondarisation à l'intérieur comme à l'extérieur du monde scientifique, en science politique comme ailleurs.

Pour terminer, il serait temps d'en finir avec la fiction qu'il n'y a qu'une condition féminine. Certes, la généralisation de la domination masculine fait de toutes les femmes des dominées, mais au-delà d'une telle trivialité, il faut voir que la domination sexuelle n'est pas le seul aspect de la domination à l'oeuvre dans nos sociétés. Avec Françoise

28. Margrit Eichler, Ibid., p. 4.

29. Ibid. 
Collin nous pourrions dire «je suis une femme mais je n'est pas une femme ${ }^{30}$, c'est-à-dire que l'identité d'une femme ne se résume pas à son identification au féminin. Cette sériation et cette réduction des dominées et dominés à des identiques procède de la logique de la domination. Dans ce sens, l'éclatement de la recherche féministe, tout comme son pendant l'éclatement des pratiques féministes, est un moyen de faire émerger cette «hétérogénéité» des femmes et de redonner une autre signification à la célèbre phrase de Simone de Beauvoir, «on ne naît pas femme, on le devient». Si le patriarcat tente de nous construire comme femmes, l'un des objectifs les plus importants des recherches féministes devrait être de nous faire émerger comme individus. Calquer nos analyses sur celles de nos homologues masculins, compléter leur information (et, dans bien des cas, leur formation) n'est certainement pas la voie royale à emprunter même si cela reste une tâche considérable et légitime. Nommer nous-mêmes, selon notre propre expérience, des aspects du réel jusque-là ignorés reste impératif pour notre affranchissement politique global.

30. Françoise Collin, «La même et les différences», Cahiers du GRIF, $\mathrm{n}^{\circ} 28,1984$. 